\title{
Éditorial
}

Ce dernier numéro de l'année paraîtra lors de la période des vœux et résolutions, c'est pourquoi j'en profite pour souhaiter une excellente année à tous nos lecteurs, nos évaluateurs et bien entendu tous les auteurs qui font de cette revue ce qu'elle est. Je n'oublie pas également nos secrétaires Corinne, Janine et Patricia et nos amies d'EDP Sciences, Agnès, Sophie et Céline.

Bonne année donc à tous

\section{Quelques nouvelles}

Notre tradition de délocalisation nous a conduit cette année pas très loin de notre port d'attache, au Service de protection radiologique des armées (SPRA) à Clamart. Mais peu importe la distance, la journée organisée par le Général Tréguier et notre collègue P. Gérasimo a été tout aussi passionnante que les précédentes, nous avons pu vérifier, une fois de plus, après l'épisode réussi de "l'uranium appauvri » (cf. journée SFRP du 27 novembre 2001), que le site de Percy était en parfaite adéquation avec notre métier, laboratoires du SPRA, service des brûlés et centre de transfusion sanguine. Je renouvelle, au nom du Comité, tous mes remerciements aux organisateurs et à ceux qui nous ont fait visiter leurs installations.

Deuxième point tout aussi important, nos correspondants étrangers; nous avons fait un point avec les anciens, et des nouveaux sont apparus. Notre « légion étrangère » se compose aujourd'hui de : L. Arranz Carrillo, M. Bailey, M. Besbes, A. Bouville, Ph. Duport, Ch. Hurtgen, E. Katz, I. Lund, Ch. Murith, X. Ortega, H. Riad, Fr. Spurny, M. Thieme et A. Wrixon. Nous leur souhaitons la bienvenue au sein de notre comité et $\mathrm{j}$ 'espère que leur arrivée apportera un nouvel élan à notre journal et que des articles nouveaux arriveront des différents pays ainsi représentés. Rappelons que nous acceptons, pour la première partie scientifique et technique, des articles en anglais et en français.

Enfin, vous trouverez dans ce numéro, la réponse à un courrier adressé peu après la création de la DGSNR et de l'IRSN à leurs responsables. Tous nos lecteurs sont ainsi informés sur ces deux organismes nouvellement créés. Je tiens à remercier leurs dirigeants qui ont bien compris tout l'intérêt de leurs réponses pour nos lecteurs.

Lors de la dernière réunion du Comité de rédaction nous avons décidé de réfléchir à deux nouvelles rubriques.

- La première, c'est décidé, s'appellera "Un peu d'histoire ». Nous y lirons de temps en temps des notes relativement brèves faisant le point sur tel ou tel aspect de notre histoire. Daniel Blanc, président du club histoire de la SFRP l'animera, mais je vous rassure, il ne sera pas seul, tout le comité est derrière lui. 
ÉDITORIAL

- Nous réfléchissons à une autre rubrique les «news du net », mais là il y a encore du travail à faire.

Enfin signalons, qu'un membre de notre société nous a écrit pour regretter la disparition de la rubrique de Jérôme Pellissier-Tanon. Nous aussi, sachez toutefois qu'il ne souhaitait plus continuer. Nous pensons à sa succession, mais avouez qu'il est difficile de lui succéder. Je tenais à vous en informer, ce n'est pas une suppression, mais bien une pause...

À très bientôt

Henri Métivier

Président du comité de la revue 